\title{
Glenoid Radius of Curvature and Humeral Head Volume Are Associated With Postoperative Dislocation After Arthroscopic Bankart Repair
}

\author{
Ravi Vaswani, M.D., Christopher Como, M.D., Mitch Fourman, M.D., \\ Andrew Wilmot, M.D., Camilo Borrero, M.D., Dharmesh Vyas, M.D., Ph.D., and \\ Albert Lin, M.D.
}

\begin{abstract}
Purpose: To measure bony morphologic parameters and identify their association with arthroscopic Bankart repair failure. Methods: This was a retrospective comparative study. The inclusion criteria were primary arthroscopic Bankart repair, no prior shoulder surgery, traumatic cause, and had a Bankart (soft tissue and bony) lesion evident on magnetic resonance imaging (MRI). The exclusion criteria were posterior labral pathology, multidirectional instability, connective tissue disorder, rotator cuff pathology, and those who underwent concomitant shoulder procedures. Patients who had a postoperative redislocation (unstable group) were compared with matched patients who did not (stable group). Data were obtained by chart review and from preoperative MRI. Comparisons were made using the Student $t$ test, Fisher exact test, or $\chi^{2}$ test. Statistical significance was defined as $P<.05$. Inter-rater reliability was measured between reviewers. Results: A total of 45 patients experienced a postoperative dislocation and were matched to 90 patients without a postoperative dislocation. There were no differences in demographic and radiographic variables. The radius of curvature (ROC) of the glenoid was larger (shallower) in the unstable group (23.6 mm vs $22.6 \mathrm{~mm}, P=.05)$. The humeral head volume (HHV) trended higher in the unstable group $(68.9 \mathrm{~mL}$ vs $62.9 \mathrm{~mL}, P=.06)$. The glenoid volume was not significantly different. A greater percentage of patients with a glenoid ROC of $24.5 \mathrm{~mm}$ or greater $(62.1 \%$ vs $26.4 \%$, $P=.0003)$ and an HHV of $80 \mathrm{~mm}^{3}$ or greater $(60.8 \%$ vs $28.9 \%, P=.003)$ experienced a redislocation compared with patients without these factors. Patients with a glenoid ROC of $24.5 \mathrm{~mm}$ or more and an HHV of $80 \mathrm{~mm}^{3}$ or more had greater than 4 times the odds of redislocation (odds ratio, 4.56; 95\% confidence interval, 1.44-14.43; $P=.0098$ ). Strong inter-rater reliability was found for the HHV, glenoid volume, glenoid ROC, and humeral head ROC measurements ( $r=0.94, r=0.88, r=0.89$, and $r=0.95$, respectively). Conclusions: This study shows that large ROC (shallow) glenoids in conjunction with large humeral heads may predispose patients to failure after arthroscopic Bankart repair. Level of Evidence: Level III, retrospective comparative trial.
\end{abstract}

$\mathbf{V}$ arious demographic, radiographic, and surgical risk factors for redislocation after arthroscopic Bankart repair have been identified. ${ }^{1}$ Despite identification of these risk factors, the long-term redislocation rate after arthroscopic Bankart repair has been reported to be as high as $30 \% .^{2}$ Variations in the bony

From the Department of Orthopaedic Surgery, University of Pittsburgh Medical Center, Pittsburgh, Pennsylvania, U.S.A. (R.V., C.C., M.F., D.V., A.L.); and Department of Radiology, University of Pittsburgh Medical Center, Pittsburgh, Pennsylvania, U.S.A. (A.W., C.B.).

The authors report the following potential conflicts of interest or sources of funding: D.V. is on the editorial board of American Journal of Sports Medicine. A.L. is a paid consultant for Arthrex and Wright Medical/Tornier. Full ICMJE author disclosure forms are available for this article online, as supplementary material.

Received April 3, 2020; accepted December 29, 2020. morphology of the glenohumeral articulation may predispose certain patients to redislocation after arthroscopic Bankart repair. The critical shoulder angle (CSA) has been shown to affect glenohumeral translation in biomechanical studies. ${ }^{3}$ Glenoid retroversion and inferior inclination have been shown to be

Address correspondence to Albert Lin, M.D., Shoulder Surgery and Sports Medicine, Freddie Fu Sports Medicine Center, University of Pittsburgh, $3200 \mathrm{~S}$ Water St, Pittsburgh, PA 15203, U.S.A.E-mail:lina2@upmc.edu

(C) 2021 THE AUTHORS. Published by Elsevier Inc. on behalf of the Arthroscopy Association of North America. This is an open access article under the CC BY-NC-ND license (http://creativecommons.org/licenses/by-nc-nd/4.0/). 2666-061X/20424

https://doi.org/10.1016/j.asmr.2020.12.010 
associated with anterior shoulder dislocation. ${ }^{4}$ These studies have focused on identifying the risk of a first-time dislocation, but the relationship between bony morphologic parameters and recurrent instability after arthroscopic Bankart repair is unknown.

Modern radiographic software allows measurement of 3-dimensional (3D) volume on magnetic resonance imaging (MRI). ${ }^{5,6}$ The ability to measure the dimensions of bony shoulder structures, including the radius of curvature (ROC), glenoid volume, and humeral head volume (HHV), based on preoperative imaging could help surgeons identify which patients may be at higher risk of postoperative dislocation. These parameters represent the morphology of the glenohumeral articulation, and anatomic variations thereof may change the tension of the soft-tissue joint stabilizers or the congruity of the articulation, predisposing some patients to dislocation even after Bankart repair.

The aim of this study was to measure bony morphologic parameters and identify their association with arthroscopic Bankart repair failure. The hypothesis was that a higher ratio of HHV to glenoid volume and a shallower glenoid socket (larger ROC) would be associated with recurrent dislocation.

\section{Methods}

Institutional review board approval was obtained prior to study initiation. Patient medical records from January 2006 to December 2015 were reviewed to identify patients who underwent primary arthroscopic Bankart repair performed by 1 of 7 surgeons at a single institution. Each surgeon performed arthroscopic shoulder stabilization using a standard suture anchor technique with a concomitant capsular shift. The positioning of patients, number of suture anchors used, use of knots, and rehabilitation protocol varied based on surgeon preference. The inclusion criteria were primary arthroscopic Bankart repair, no prior shoulder surgery, traumatic cause, and had a Bankart (soft tissue and bony) lesion evident on MRI. The exclusion criteria were posterior labral pathology, multidirectional instability, connective tissue disorder, rotator cuff pathology, and those who underwent concomitant shoulder procedures. Patients with a recurrent instability event postoperatively, defined as a dislocation or subluxation, and met the inclusion criteria were classified as having surgical failure (unstable group). Whether the patients experienced a dislocation event was determined by chart review of the office notes. The patients in the unstable group were matched to a cohort of patients who underwent primary arthroscopic Bankart repair during the same period but did not experience postoperative dislocation and who met the inclusion criteria (stable group). The groups were matched for age and sex because these variables have previously been shown to be postoperative dislocation risk factors. ${ }^{7}$ This matched cohort was chosen to explore the variables specific to this population that predispose patients to postoperative dislocation. Patients who were not included in this study either did not meet the inclusion criteria or were not matched, in terms of age and sex, with patients in the unstable group.

The primary outcomes collected were the HHV, glenoid volume, and ROC. Demographic data collected included sex, age at the time of surgery, mechanism of injury, sport played, whether the patient was a contact athlete, whether the patient was an overhead or throwing athlete, level of sports participation, length of follow-up, number of prior dislocations, and whether the patient was cleared for sports participation at final follow-up. Surgical variables assessed included number of anchors used and surgical positioning. Two musculoskeletal imaging-trained radiologists (A.W. and C.B.) analyzed preoperative radiographs and MRI scans and were blinded to whether the patients experienced any postoperative instability. Radiographic parameters collected included glenoid bone loss; whether HillSachs lesions were on or off track; and the presence of a glenoid labrum articular defect (GLAD), anterior labral periosteal sleeve avulsion (ALPSA), Perthes lesion, or humeral avulsion of the glenohumeral ligament (HAGL).

The volume and ROC of the humeral head and glenoid were measured using Vitrea software (Vital Images, Minnetonka, MN). This proprietary software allows the user to outline a structure in each plane of view (axial, coronal, and sagittal) and measure its volume by constructing a 3D volume from magnetic resonance images (Fig 1). For this study, the axial T2 MRI sequence was loaded in Vitrea's advanced viewer. The length of the coracoid process was measured using the ruler tool in Vitrea, starting at the base and extending to the tip of the coracoid. For the HHV and glenoid volume, the borders of each structure were outlined on the axial and reconstructed coronal and sagittal images. The distal extent of the humeral head was defined as the surgical humeral neck. The medial extent of the glenoid was defined as the location where the glenoid neck met the scapular spine. A 3D model of the structure was then constructed, and the volume was measured. For the ROC, the user placed a best-fit circle on the humeral head and glenoid, and the radius was measured from the periphery of the circle to the center (Fig 2).

Statistical analysis was performed using the Student $t$ test for continuous variables and the Fisher exact test or $\chi^{2}$ test for categorical variables. Logistic regression analysis was used to compute adjusted odds ratios (ORs). Inter-rater reliability was calculated between 2 reviewers' (A. W. and C. B.) measurements of the radiographic variables using the interclass correlation coefficient $\operatorname{ICC}(3,1)$ formula of Shrout and Fleiss. 
Fig 1. Humeral head volume measurement in Vitrea. The axial Tl image of a right shoulder is loaded in the Vitrea software, which then generates reconstructed sagittal and coronal images (indicated by the red areas highlighted). The sculpt tool is selected, and the humeral head is traced on the axial, sagittal, and coronal images. This generates a 3-dimensional model (bottom left corner), and the volume of this model is measured.

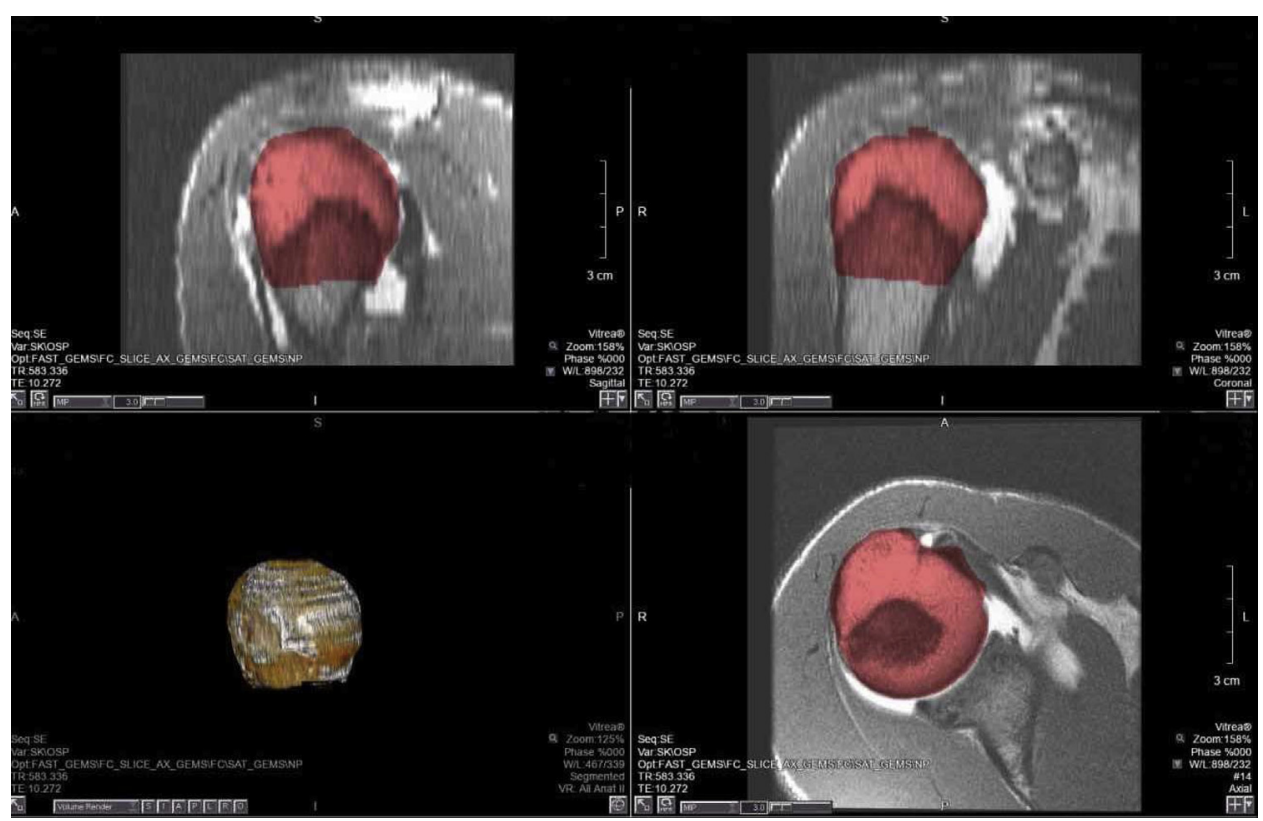

Interclass correlation coefficients ${ }^{8}$ were graded as excellent $(\geq 0.75)$, good (0.60-0.74), fair (0.40-0.59), or poor $(<0.40)$. Statistical significance was set at .05 , and all tests were 2 -sided.

\section{Results}

In the electronic record, 289 patients who underwent Bankart repair were identified, of whom 45 (15.6\%) experienced a postoperative dislocation event and met the inclusion criteria. This unstable group was matched (based on age and sex) to a cohort of 90 patients with stable shoulders who did not experience a postoperative dislocation event and met the inclusion criteria. We found no significant difference between the unstable and stable groups in age at the time of surgery (18.6 years vs 18.6 years, $P=.95)$, sex $(71 \%$ male patients vs $71 \%$ male patients, $P>.99)$, participation in contact sports $(67 \%$ vs $64 \%, P=.79)$, percentage of throwing athletes $(13 \%$ vs $10 \%, P=.57)$, or percentage of overhead athletes ( $22 \%$ vs $13 \%, P=.13)$ (Table 1$)$. Mechanisms of injury in the stable group consisted of sports injury in $75 \%$ of patients, falls in $21 \%$, and other trauma in $4 \%$, whereas in the unstable group, $71 \%$ of patients had sports injury and $29 \%$ had falls. The distribution of primary sport played as well as the level of participation for each group is shown in Table 2. The average follow-up period was 28 months (range, 4-75 months) in the unstable group and 8 months (range, 260 months $)$ in the stable group $(P<.0001)$. The average time to postoperative dislocation in the unstable group was 17 months (range, 2.2-67.2 months). A significantly higher proportion of patients in the stable group were cleared for return to sport after surgery at the time of final follow-up than in the unstable group
(96\% vs $64 \%, P<.0001$ ). We observed no differences between the unstable and stable groups among the surgical variables, including the proportion of patients in the lateral decubitus position ( $24 \%$ vs $38 \%, P=.17)$ and the median number of suture anchors used (4 [range, 1-6] vs 3.5 [range, 1-6]; $P=.74$ ). The average number of preoperative dislocations was significantly higher in the unstable group ( 3.1 vs $1.9, P=.008$ ) (Table 1).

In terms of radiographic parameters, the unstable and stable groups had similar percentages of patients with

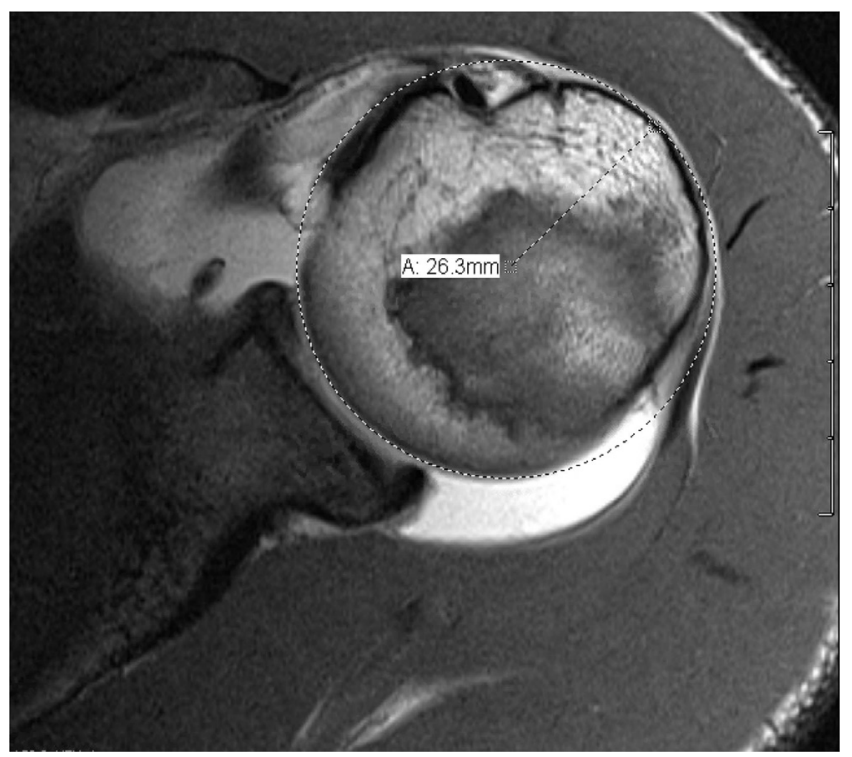

Fig 2. Glenoid radius-of-curvature measurement: axial $\mathrm{Tl}$ image of left shoulder. The circle tool is used to create a bestfit circle that matches the curvature of the glenoid. Then, the measure tool is used to measure the radius of this circle. 
Table 1. Comparison of Demographic Data

\begin{tabular}{lccc}
\hline & Unstable & Stable & $P$ Value \\
\hline Age, mean \pm SD, yr & $18.6 \pm 3.1$ & $18.6 \pm 4.0$ & .95 \\
Male patients, $\mathrm{n}(\%)$ & $32(71)$ & $64(71)$ & $>.99$ \\
$\begin{array}{l}\text { Contact sports } \\
\quad \text { participation, n (\%) }\end{array}$ & $30(67)$ & $58(64)$ & .79 \\
$\begin{array}{l}\text { Throwing athletes, n (\%) } \\
\text { Overhead athletes, n (\%) }\end{array}$ & $6(13)$ & $9(10)$ & .57 \\
$\begin{array}{l}\text { Median No. of anchors } \\
\quad \text { during repair }\end{array}$ & $4 \pm 0.9$ & $3.5 \pm 1.0$ & .74 \\
$\begin{array}{l}\text { Preoperative dislocations, } \\
\quad \text { mean } \pm \text { SD }\end{array}$ & $3.1 \pm 2.8$ & $1.9 \pm 1.4$ & .008 \\
\hline
\end{tabular}

SD, standard deviation.

GLAD, ALPSA, Perthes, HAGL, and off-track Hill-Sachs lesions, as well as similar glenoid bone loss (Table 3 ). Glenoid version $\left(-6.3^{\circ}\right.$ vs $\left.-6.8^{\circ}, P=.59\right)$, glenoid inclination $\left(11.9^{\circ}\right.$ vs $\left.12.4^{\circ}, P=.75\right)$, and the CSA $\left(32.8^{\circ}\right.$ vs $\left.33.3^{\circ}, P=.56\right)$ did not differ between groups. The glenoid volume ( $13.5 \mathrm{~mL}$ vs $12.8 \mathrm{~mL}, P=.31$ ), ratio of HHV to glenoid volume (5.2 vs 5.0, $P=.20$ ), humeral head ROC (26.2 $\mathrm{mm}$ vs $25.6 \mathrm{~mm}, P=.22)$, and coracoid length (27.7 $\mathrm{mm}$ vs $27.4 \mathrm{~mm}, P=.58$ ) were also similar between the 2 groups. The HHV $(68.9 \mathrm{~mL}$ vs $62.9 \mathrm{~mL}, P=.06)$ and ROC of the glenoid $(23.6 \mathrm{~mm}$ vs $22.6 \mathrm{~mm}, P=.05$ ) were larger in the unstable group; these differences approached-but did not reach-statistical significance.

A higher proportion of patients with a glenoid ROC of $24.5 \mathrm{~mm}$ or greater exhibited postoperative instability when compared with those with a glenoid ROC of less than $24.5 \mathrm{~mm}(62.1 \%$ vs $26.4 \%, P=.0003)$. There was also a higher proportion of patients with an HHV of 80 $\mathrm{mm}^{3}$ or greater who had a postoperative dislocation compared with those with an HHV of less than $80 \mathrm{~mm}^{3}$ $(60.8 \%$ vs $28.9 \%, P=.003)$. Patients with the combination of a glenoid ROC of $24.5 \mathrm{~mm}$ or greater and an HHV of $80 \mathrm{~mm}^{3}$ or greater had over 4 times the

Table 2. Comparison of Sports Played and Level of Sports Participation

\begin{tabular}{lccc}
\hline & Unstable & Stable & $P$ Value \\
\hline Sport played, n (\%) & $18(40)$ & $38(42)$ & .20 \\
Football & $9(20)$ & $11(12)$ & \\
Wrestling & $4(9)$ & $9(10)$ & \\
Basketball & $3(7)$ & $4(4)$ \\
Soccer & $3(7)$ & $2(2)$ \\
Softball & $3(7)$ & $2(2)$ \\
Volleyball & $1(2)$ & $0(0)$ \\
Lacrosse & $2(4)$ & $7(8)$ \\
Hockey & $1(2)$ & $3(3)$ \\
Baseball & $1(2)$ & $14(16)$ \\
Other & & \\
Level of sports participation, \% & 22 & 20 \\
College & 51 & 64 \\
High school & 3 & 1 \\
Middle school & 22 & 15 \\
Recreational & & \\
\hline
\end{tabular}

odds of postoperative instability (OR, 4.56; 95\% confidence interval $[\mathrm{CI}], 1.44-14.43 ; P=.0098$ ) compared with those with an ROC of less than $24.5 \mathrm{~mm}$ and HHV of less than $80 \mathrm{~mm}^{3}$. On further analysis, there were no increased odds of a postoperative dislocation with only one of these factors, either a glenoid ROC of $24.5 \mathrm{~mm}$ or greater alone (with an HHV $<80 \mathrm{~mm}^{3}$ ) (OR, 1.5; $95 \%$ CI, $0.33-6.77 ; P=.60)$ or an HHV of $80 \mathrm{~mm}^{3}$ or greater alone (with a glenoid ROC $<24.5 \mathrm{~mm}$ ) (OR, 2.0; 95\% CI, 0.35-11.54; $P=.44)$. No difference was found in the number of preoperative dislocations in patients with larger versus smaller glenoid ROCs (2.6 vs $2.3, P=.55)$.

Logistic regression analysis showed an increasing odds of postoperative dislocation with an increasing glenoid ROC (OR, 21.91; 95\% CI, 1.98-303.2), which was independent of other variables, including the number of preoperative dislocations. The receiver operating curve for a glenoid ROC of $24.5 \mathrm{~mm}$ or greater showed an area under the curve of 0.68 (95\% CI, 0.57-0.79; $P=.0015)$, indicating good specificity and sensitivity for predicting postoperative dislocation (Fig 3). Inter-rater reliability between 2 independent reviewers was excellent for the measurements of the HHV $(r=0.94)$, glenoid volume $(r=0.88)$, ROC of the glenoid $(r=0.89)$, and ROC of the humeral head $(r=0.95)$.

\section{Discussion}

The major finding of this study was that a larger glenoid ROC, corresponding to a shallower glenoid, and a larger HHV were associated with recurrent dislocation after arthroscopic Bankart repair, which confirmed part of our hypothesis. The ratio of HHV to glenoid volume was not correlated with recurrent dislocation. In addition, the combination of an HHV of $80 \mathrm{~mm}^{3}$ or greater and glenoid ROC of $24.5 \mathrm{~mm}$ or greater was associated with a 4 -fold increase in risk regarding postoperative dislocation. The method of bony morphologic measurement presented in this study was also found to have high inter-rater reliability. Therefore, the use of 3D software to measure bony morphologic parameters is not only reproducible but also useful for predicting postoperative failure.

Table 3. Comparison of Radiographic Parameters

\begin{tabular}{lccc}
\hline & Unstable & Stable & $P$ Value \\
\hline GLAD, n (\%) & $8(19)$ & $18(24)$ & .47 \\
ALPSA, n (\%) & $11(26)$ & $15(20)$ & .48 \\
Perthes lesion, n (\%) & $12(29)$ & $32(44)$ & .13 \\
HAGL, n (\%) & $1(2)$ & $0(0)$ & .36 \\
Off-track Hill-Sachs lesion, n (\%) & $2(6)$ & $4(7)$ & .94 \\
Glenoid bone loss, \% & 1 & 3 & .43 \\
\hline
\end{tabular}

ALPSA, anterior labral periosteal sleeve avulsion; GLAD, glenoid labrum articular defect; HAGL, humeral avulsion of glenohumeral ligament. 


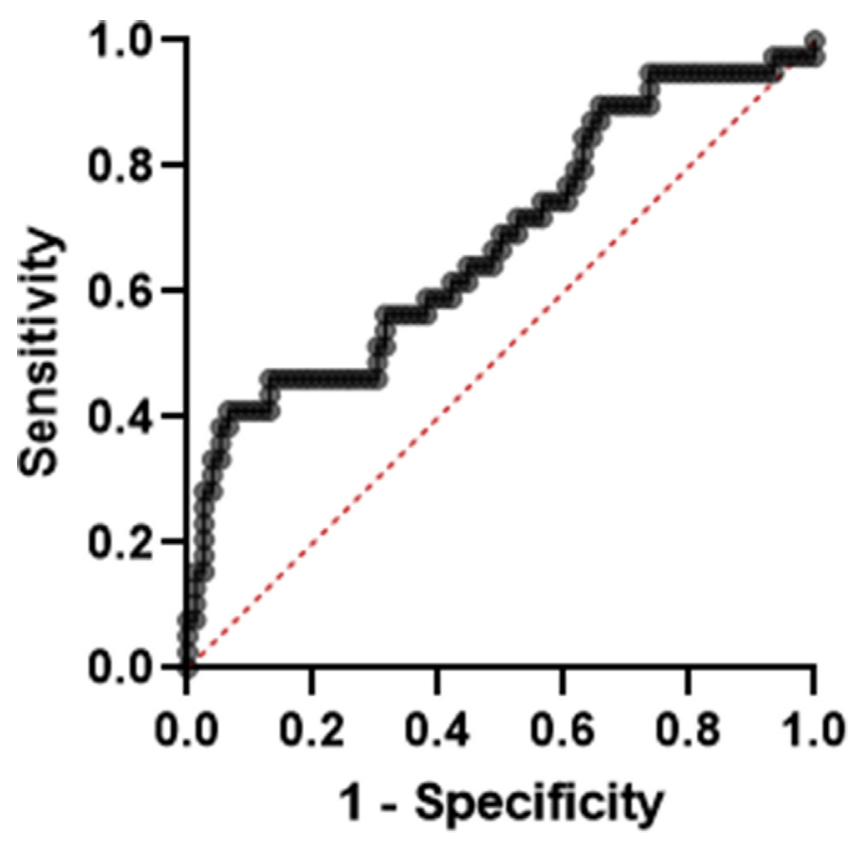

Fig 3. Receiver operating curve for glenoid radius of curvature.

The glenohumeral joint is an inherently unstable joint that relies on soft-tissue stabilizers to keep the humeral head seated in the glenoid, and it is often described as a "golf ball on a tee" owing to the large nature of the humeral head relative to the shallow glenoid. Because of the reliance on soft-tissue structures, slight variations in normal anatomy may predispose patients to recurrent shoulder instability. Arthroscopic Bankart repair aims to restore and augment the native anatomy of the capsulolabral complex and correct pathologic lesions such as ALPSA ${ }^{9}$ or HAGL lesions. ${ }^{10}$ Long-term results have shown high rates of recurrent instability and have remained relatively consistent over 2 decades despite improvements in arthroscopic techniques, ${ }^{1,2,11}$ suggesting that the causes of recurrence are not well understood.

Variations in bony morphology are recognized as important contributors to certain conditions. An increased CSA, for example, has been found to correlate with the presence of a rotator cuff tear, whereas a decreased CSA is associated with osteoarthritis. ${ }^{12}$ Zaid et al. ${ }^{13}$ reviewed the anatomic parameters that have been studied in the literature in relation to rotator cuff tears and found that an increased CSA, increased acromial index, and decreased lateral acromial angle were associated with rotator cuff tears. Glenoid dysplasia, characterized by hypoplasia and retroversion, has been linked to the development of premature osteoarthritis and shoulder instability. ${ }^{14}$

Glenoid version has also been linked to shoulder instability. Hohmann and Tetsworth ${ }^{4}$ compared patients with versus without a history of shoulder instability and found that the instability group had more glenoid anteversion by $4.1^{\circ}$ and inferior inclination by $2.4^{\circ}$. Aygün et al. ${ }^{15}$ similarly found that dislocated shoulders had greater glenoid anteversion than stable shoulders by $4.2^{\circ}$. Eichinger et al. ${ }^{16}$ performed a biomechanical study examining the effects of varying degrees of version on the force needed to dislocate a cadaveric shoulder. They found that with increasing anteversion, the anterior dislocation force decreased significantly, and with increasing retroversion, the posterior dislocation force decreased significantly. Increased glenoid anteversion has also been associated with arthropathy after shoulder instability. ${ }^{17}$ Although this relation has been established for primary shoulder dislocation, the effect of glenoid anteversion and inferior inclination on recurrent dislocation after arthroscopic Bankart repair has not been studied in the literature. In our study, no difference in glenoid version or inclination was found between the recurrent dislocation and stable groups. Moreover, the CSA was not associated with postoperative dislocation.

Studies have also shown that a shallow glenoid may predispose patients to shoulder dislocation. Peltz et al. ${ }^{18}$ studied the effect of the glenoid ROC on primary shoulder dislocation. They found that shoulders that experienced a dislocation had larger ROCs than shoulders that had not. They posited that this finding was due to the shallower morphology of the unstable glenoids. Moroder et al. ${ }^{19}$ identified an association between flattening of the bony glenoid concavity and recurrent instability. Therefore, glenoid morphology clearly plays an important role regarding shoulder stability. Whether shallow glenoid morphology influences postoperative instability after arthroscopic Bankart repair has not been as well studied.

The aforementioned studies imply that evaluation of a failed shoulder instability procedure should include consideration of the patient's bony anatomy. Although the soft-tissue structures are frequently injured during a shoulder dislocation, the underlying abnormality may be the variation in the bony anatomy. Therefore, although arthroscopic Bankart repair typically includes some degree of capsular plication to augment the softtissue stabilizers, this may not be enough to overcome the patient's baseline predisposition to shoulder instability.

In our study, the combination of an HHV of $80 \mathrm{~mm}^{3}$ or greater and a glenoid ROC of $24.5 \mathrm{~mm}$ or greater was associated with recurrent dislocation after arthroscopic Bankart repair. Logistic regression analysis found that a larger glenoid ROC was associated with dislocation independent of other variables, although the CI was wide, indicating that a larger sample size is required to find a more exact CI. The threshold value of $24.5 \mathrm{~mm}$ was chosen based on analysis of the data and experiential opinion to indicate a clinically usable value that would 
optimize the positive predictive value and sensitivity. The receiver operating curve for the glenoid ROC indicates that it can be used as a tool to identify patients who are at higher risk of dislocation postoperatively.

A larger HHV may predispose to shoulder dislocation by decreasing the contact area of the glenohumeral articulation relative to the whole surface area of the humeral head or by increasing the range of motion of glenohumeral joint, thus placing the soft-tissue stabilizers at risk of failure at the extremes of motion. Furthermore, because the glenohumeral articulation relies on these soft-tissue stabilizers, a larger HHV would place them at risk of injury owing to the increased momentum generated during range of motion. A larger glenoid ROC represents a shallow glenoid morphology, which may confer even less stability of the glenohumeral articulation compared with the already inherently unstable normal glenohumeral joint. Patients who had a shallower glenoid morphology (ROC $\geq 24.5 \mathrm{~mm})$ and a larger HHV $\left(\geq 80 \mathrm{~mm}^{3}\right)$ had 4 -fold higher odds of experiencing a postoperative dislocation compared with those who had neither. Therefore, identification of these patients who are predisposed to postoperative instability may be important before indicating patients for arthroscopic shoulder stabilization.

Furthermore, the existing measurements of glenoid concavity depth in the current literature are based on computed tomography scans, which are not frequently obtained preoperatively. ${ }^{18,19}$ The method presented in our study uses preoperative MRI to measure the ROC, which is often the preferred imaging modality in patients with shoulder instability, particularly those in whom there is no suspicion of significant glenoid or humeral bone loss. The use of MRI also allows the user to measure the $3 \mathrm{D}$ volume of the bony structures. The inter-rater reliability was high for most bony morphologic measures, which shows that this method could likely be adopted by many surgeons.

The glenoid volume was not significantly different between the unstable and stable groups. This was likely due to the fact that the surface anatomy of the glenoid is more important to glenohumeral stability than the volume. Likewise, the ratio of HHV to glenoid volume was not significantly different between the groups, given that this also does not account for the variations in surface anatomy of the glenoid. Furthermore, the humeral head ROC was similar between the groups. This was likely due to the fact that the ROC of the humeral head does not account for the effect of a larger HHV, which plays a greater role regarding tension of the soft-tissue stabilizers around the joint than the ROC.

The main strength of this study is the introduction of an accurate and reproducible method of evaluating bony morphologic parameters on preoperative MRI. This method can be used by all surgeons to individualize the treatment plan for each patient. In addition, the specific parameters of glenoid ROC and HHV may help identify individuals who are at risk of failure after arthroscopic Bankart repair and may be more appropriately indicated for other stabilization procedures. Future studies should focus on identifying surgical options for patients with a large glenoid ROC to determine if additional augmentation or other procedures can decrease recurrent dislocations postoperatively.

\section{Limitations}

The limitations of this study include the potential for selection bias with surgical decision making owing to the retrospective design and the inability to draw conclusions about causality owing to the case-control study construct. The average follow-up period was also longer in the unstable group, which may be because of the need for continued treatment after recurrent postoperative dislocation and the loss to follow-up of patients in the stable group who are doing well. The shorter follow-up period in the stable group, however, could also mean that there may be more patients who experience a postoperative dislocation with longer follow-up. Another limitation is the lack of an a priori power analysis. A post hoc analysis determined that the inclusion of 396 subjects would be required to show a difference between groups. We believe that this would not be feasible for this type of study to obtain adequate follow-up to identify postoperative dislocations.

\section{Conclusions}

This study shows that large ROC (shallow) glenoids in conjunction with large humeral heads may predispose patients to failure after arthroscopic Bankart repair.

\section{References}

1. Donohue MA, Mauntel TC, Dickens JF. Recurrent shoulder instability after primary Bankart repair. Sports Med Arthrosc 2017;25:123-130.

2. Flinkkilä T, Knape R, Sirniö K, Ohtonen P, Leppilahti J. Long-term results of arthroscopic Bankart repair: Minimum 10 years of follow-up. Knee Surg Sport Traumatol Arthrosc 2018;26:94-99.

3. Moor BK, Kuster R, Osterhoff G, et al. Inclinationdependent changes of the critical shoulder angle significantly influence superior glenohumeral joint stability. Clin Biomech (Bristol, Avon) 2016;32:268-273.

4. Hohmann E, Tetsworth K. Glenoid version and inclination are risk factors for anterior shoulder dislocation. J Shoulder Elbow Surg 2015;24:1268-1273.

5. Pattanayak P, Turkbey EB, Summers RM. Comparative evaluation of three software packages for liver and spleen 
segmentation and volumetry. Acad Radiol 2017;24: 831-839.

6. Paredes JL, Orabi AI, Ahmad T, et al. A non-invasive method of quantifying pancreatic volume in mice using micro-MRI. PLoS One 2014;9:e92263.

7. Balg F, Boileau P. The Instability Severity Index score: A simple pre-operative score to select patients for arthroscopic or open shoulder stabilisation. J Bone Joint Surg Br 2007;89:1470- 1477 .

8. Kuhnen AH, Marcello PW, Roberts PL, et al. Can the National Surgical Quality Improvement Program provide surgeonspecific outcomes? Dis Colon Rectum 2015;58:247-253.

9. Park JY, Lee SJ, Lhee SH, Oh JH. Change in labrum height after arthroscopic Bankart repair: Correlation with preoperative tissue quality and clinical outcome. J Shoulder Elbow Surg 2012;21:1712-1720.

10. George MS, Khazzam M, Kuhn JE. Humeral avulsion of glenohumeral ligaments. J Am Acad Orthop Surg 201 1;19: 127-133.

11. Aboalata M, Plath JE, Seppel G, Juretzko J, Vogt S, Imhoff AB. Results of arthroscopic Bankart repair for anterior-inferior shoulder instability at 13-year follow-up. Am J Sports Med 2017;45:782-787.

12. Spiegl UJ, Horan MP, Smith SW, Ho CP, Millett PJ. The critical shoulder angle is associated with rotator cuff tears and shoulder osteoarthritis and is better assessed with radiographs over MRI. Knee Surg Sport Traumatol Arthrosc 2016;24:2244-2251.
13. Zaid MB, Young NM, Pedoia V, Feeley BT, Ma CB, Lansdown DA. Anatomic shoulder parameters and their relationship to the presence of degenerative rotator cuff tears and glenohumeral osteoarthritis: A systematic review and meta-analysis. J Shoulder Elbow Surg 2019;28:2457-2466.

14. Abboud JA, Bateman DK, Barlow J. Glenoid dysplasia. J Am Acad Orthop Surg 2016;24:327-336.

15. Aygün Ü, Çalik Y, Işik C, Şahin H, Şahin R, Aygün DÖ. The importance of glenoid version in patients with anterior dislocation of the shoulder. J Shoulder Elbow Surg 2016;25:1930-1936.

16. Eichinger JK, Massimini DF, Kim J, Higgins LD. Biomechanical evaluation of glenoid version and dislocation direction on the influence of anterior shoulder instability and development of Hill-Sachs lesions. Am J Sports Med 2016;44:2792-2799.

17. Haas M, Plachel F, Wierer G, et al. Glenoid morphology is associated with the development of instability arthropathy. J Shoulder Elbow Surg 2019;28:893-899.

18. Peltz CD, Zauel R, Ramo N, Mehran N, Moutzouros V, Bey MJ. Differences in glenohumeral joint morphology between patients with anterior shoulder instability and healthy, uninjured volunteers. J Shoulder Elbow Surg 2015;24:1014-1020.

19. Moroder P, Ernstbrunner L, Pomwenger W, et al. Anterior shoulder instability is associated with an underlying deficiency of the bony glenoid concavity. Arthroscopy 2015;31:1223-1231. 